\title{
Specify if Other Cytogenetic Analysis Abnormality Type
}

National Cancer Institute

\section{Source}

National Cancer Institute. Specify if Other Cytogenetic Analysis Abnormality Type. NCI

Thesaurus. Code C160762.

A directive to specify if other cytogenetic analysis abnormalities were present. 